ISAHP Article: Malik, et al/Strategic Planning in Crisis Situation To Be Submitted to the International Symposium of the Analytic Hierarchy Process 2014, Washington D.C., U.S.A.

\title{
STRATEGIC PLANNING IN CRISIS SITUATION
}

\author{
G S Malik* \\ E-mail: gurinder.malik@gmail.com \\ Rajiv Gupta* \\ E-mail: rajiv@issa.drdo.in \\ Arun Dayal* \\ E-mail: adayal@issa.drdo.in \\ Varun Kumar Singh* \\ E-mail: vksingh@issa.drdo.in \\ *Institute for Systems Studies and Analyses, \\ Metcalfe House Complex, DRDO, \\ Delhi, INDIA
}

\begin{abstract}
In strategic planning on defence issues of a nation, methods for exploring merits, demerits of various alternative actions, strategies, counter-strategies, proposed to handle crisis situations plays an important role in the decision making process. The decision maker has to make decisions, keeping in mind several relevant factors, such as military strength, international affairs, socio-economy effects and relevant government policies. This paper proposes a methodology to analyze a mixed set of strategies for handling crisis situations in politico-military situations, using Dependency Structure Matrix (DSM) and Analytical Hierarchy Process (AHP). In this paper we consider tension/ threat index, as a metric, to understand the overall impact of various options in the decision making process. It has been defined as a quantified measure to evaluate the effect of strategy in the crisis situation on three dimensions, viz., Military Force Potential, Diplomatic standing in the world and underlying Government Policies. Analytical Hierarchy Process has been used to analyze various strategies, proposed by the experts, in the above three dimensions. Various strategies are compared with respect to the impact on different factors and sub-factors, to understand expected payoff, benefit of each of them, in handling the crisis situation. Dependency Structure Matrix (DSM) is used to capture the relationships and mutual dependencies of these strategies in the overall governance of the nation. A methodology is defined to evolve a best set of possible mixed strategies/ actions to handle the crisis situation. As a case study, the proposed methodology is applied for conducting seminar/ crisis games in analysis of two nation conflict scenarios.
\end{abstract}

Keywords: DSM, Crisis Gaming, AHP, Expected Payoff. 
ISAHP Article: Malik, et al/Strategic Planning in Crisis Situation To Be Submitted to the International Symposium of the Analytic Hierarchy Process 2014, Washington D.C., U.S.A.

\section{Introduction}

Every nation is facing some crisis situations that has a direct or indirect influence on its short or long term national interest. These crisis issues have relevance to various aspects of national interests, relating to its military, economy, polity or even on international relationships. Handling of a crisis situation is of a paramount importance for a nation. In the current environment there can be diversity of opinion to handle the crisis, but little consensus on the outcomes of the policy choice. Use of scientific ways to explore possibilities of weighting various alternatives is essential. It is important that these potential solutions are based on the best available intelligence, but are still hypothetical to the extent that not all conditions can be predicted. Even if all variations of a conflict situation can be anticipated, planning for all cannot be done feasibly.

In order to handle such multi-criteria decision making issues, an AHP based framework, supplemented with the concept of Dependency Structure Matrix (DSM) [Bhushan and Rai, 2004] is proposed for crisis management. This process can evaluate the effect of various actions, strategies, counter-strategies using threat/ tension index as a metric, in three dimensions mainly military, diplomatic and government policy.

\section{Literature Review}

A number of crisis event such as firing across Line of Control, covert war, terrorist attack at some important places or buildings, acquisition of nuclear weapons, anger of masses with in country on social, economic or military issues, etc. occurs in various parts of the world which in terms either escalate or de-escalate the tension of a nation. Strategic planning is a process composed of two phases: namely mitigation and preparedness. Mitigation is a set of strategic actions taken to reduce the effect of a crisis. Preparedness basically tactical measures aimed to reduce the happening or avoid crisis by preparing the nation for such crisis.

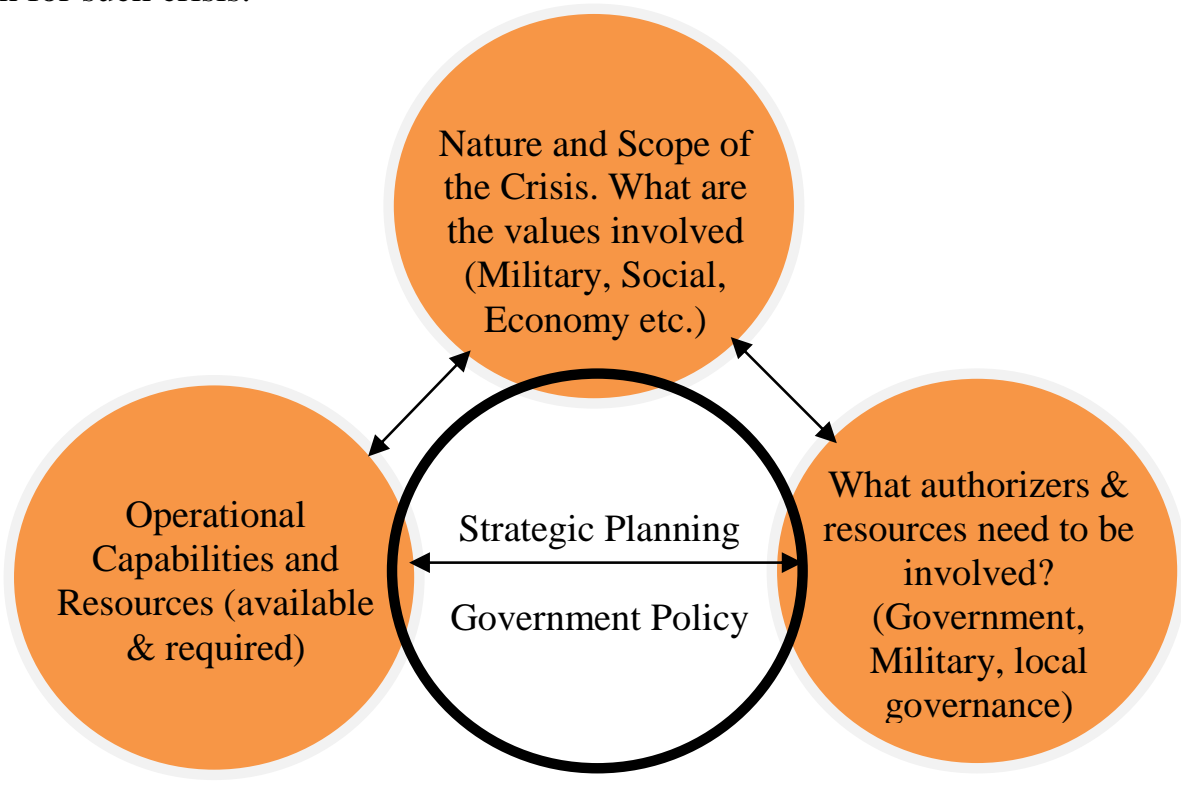

Strategic Planning on Crisis 
ISAHP Article: Malik, et al/Strategic Planning in Crisis Situation To Be Submitted to the International Symposium of the Analytic Hierarchy Process 2014, Washington D.C., U.S.A.

Previous research in strategic planning is mainly dedicated to studying the various possible courses of actions or strategies to handle a crisis in form of seminar game or free form game. A key activity in strategic planning is preparation for crisis. If the right safety measures are implemented beforehand, harmful effects can be significantly mitigated

\section{Research Design/Methodology}

The impact of proposed actions, strategies, counter-strategies on different aspects of national resources, namely military force potential, diplomatic standing, economic strengths, internal polity \& well-being, etc., are a key to success in any crisis situation. The quantified indices can have both positive and negative aspects. On one hand certain actions may impose burden on economy, unrest in internal polity, criticism from international community, it may on the other hand reflect growth in trade, enhancement of military potential, diplomatic support from strong world powers, etc. The two aspects described above have two clear aspects of analysis. On one hand we need to identify alternation actions, strategies, counter strategies for handling politico-military crisis situations, evaluate their merits, demerits and grade them for prioritized execution. On the other hand, execution of certain strategies may impact certain other aspects and therefore their analysis of merits, demerits have also consider mutual dependencies, before grading them for prioritized execution. The problem is complex and requires inputs from experts from different domain namely military, Diplomatic and government policy. These are often qualitative and there is a very complex dependency among the alternatives from various domains.

The various crisis scenario affecting the national security are first ascertained in detail. For a crisis situation the various factors and subfactor to define the AHP framework are defined. The experts from different domains are asked to provide various strategies which may prove beneficial(reduce the tension). These strategies are then prioritized based on the AHP framework. Since the strategies are from different domains the interdependencies are captured using Dependency Structure matrix(DSM). These matrices generate the Decision Impact Estimator (DIE) for the various strategies. Using the Benefit values derived from the AHP Hierarchy and the Decision Impact Estimator the set of strategies are evaluated.

\section{Data/Model Analysis}

One of the biggest strategic challenge of modern times is combating terrorism. Any terrorist attack leads to a crisis situation which threatens the foundation of any nation. The growing menace has become major challenge to world stability and it is of paramount importance to respond to terrorism at all levels and in all dimensions. How the nation fights the terrorist organization may impact on its own long - term stability.

An AHP framework is used to prioritise and evaluate the quantitative benefit of the proposed strategies against the crisis scenario. The problem is analysed from different domains of military, diplomacy and government policy and benefits of different proposed strategies in terms of reduction of tension is captured from different domain experts. 
ISAHP Article: Malik, et al/Strategic Planning in Crisis Situation To Be Submitted to the International Symposium of the Analytic Hierarchy Process 2014, Washington D.C., U.S.A.

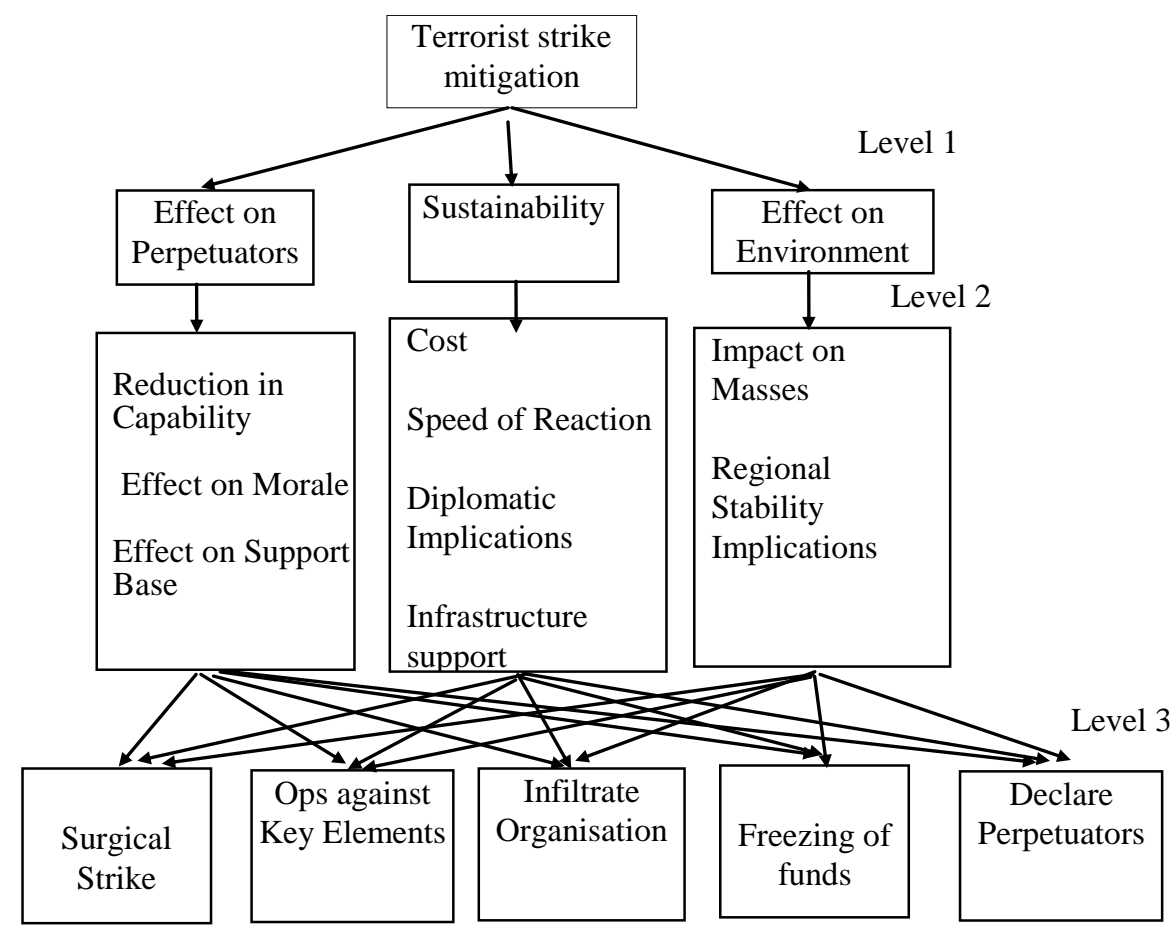

Since the decisions are from multiple domains the inherent dependencies or, synergistic effects and complex relationships among the proposed solutions needs to be evaluated. Dependency Structure matrix is used to capture the complex inter relationships of the proposed strategies in terms of No dependency low, medium, high and extreme dependency.

\begin{tabular}{|c|c|c|c|c|c|c|c|}
\hline Strategies & & A1 & A2 & A3 & A4 & A5 & A6 \\
\hline $\begin{array}{c}\text { Surgical } \\
\text { Strike }\end{array}$ & A1 & 1 & high & No & No & Medium & High \\
\hline $\begin{array}{c}\text { Ops against } \\
\text { key elements }\end{array}$ & A2 & No & 1 & No & No & Medium & High \\
\hline $\begin{array}{c}\text { Infiltrate } \\
\text { Orgn }\end{array}$ & A3 & No & No & 1 & No & No & Low \\
\hline $\begin{array}{c}\text { Freezing of } \\
\text { funds }\end{array}$ & A4 & No & No & No & 1 & No & No \\
\hline $\begin{array}{c}\text { Declare } \\
\text { Perpetuators }\end{array}$ & A5 & No & No & No & No & 1 & Medium \\
\hline $\begin{array}{c}\text { Govt } \\
\text { commitment } \\
\text { for fitting } \\
\text { response }\end{array}$ & A6 & No & No & No & No & No & 1 \\
\hline
\end{tabular}


ISAHP Article: Malik, et al/Strategic Planning in Crisis Situation To Be Submitted to the International Symposium of the Analytic Hierarchy Process 2014, Washington D.C., U.S.A.

The above DSM matrix is used to evaluate a Decision Impact Estimator(DIE) which is the measure of a strategy complexity measure. This is combined with the AHP frame work values to ascertain the best mix of strategy for handling the situation.

\section{Limitations}

The analysis proposed above requires good deal of understanding of all aspects of politico-military-eco-social issues. Experts from top bureaucracy of the nation can only provide such information. Such information can also be generated through seminars, meetings, repeated and aggregated over large varied participating experts to generate a meaningful data set. The data set may have to be revised in course of time to suit changing situations.

\section{Conclusions}

The process outlined above has rich potential for handling strategic planning issues. It attempts to quantify complex decisions when these decisions are from cross domains and are complexly interlinked. This methodology is suitable for automating complex decision making.

\section{Key References}

Bhushan, N., \& Rai, K. (2004). Strategic Decision Making - Applying the Analytic Hierarchy Process, Springer, UK.

Geckil I. K. \& Anderson P. L. (2010). Applied Game and Strategic Behaviour, Chapman $\&$ Hall/CRC Press.

\section{Appendices}

Occasionally, you may want to include appendices such as a survey sample (if data was collected via survey), a picture (e.g. a particular geographical site), etc. Appendices are not needed but if you believe they can improve the readability of your submission, they can be included here provided the total number of pages for your submission does not go above the limit indicate in the first page (short abstract). 